\title{
Transcranial Magnetic Stimulation of Frontal Oculomotor Regions during Smooth Pursuit
}

\author{
Danny Gagnon, ${ }^{1}$ Tomás̆ Paus, ${ }^{1,3}$ Marie-Helène Grosbras, ${ }^{3}$ G. Bruce Pike, ${ }^{4}$ and Gillian A. 0 'Driscoll $1,2,4$ \\ Departments of ${ }^{1}$ Psychology and ${ }^{2}$ Psychiatry, McGill University, and ${ }^{3}$ Cognitive Neuroscience Unit and ${ }^{4} \mathrm{McC}$ Connell Brain Imaging Center, Montreal \\ Neurological Institute, McGill University, Montreal, Canada H3A 1B1
}

Both the frontal eye fields (FEFs) and supplementary eye fields (SEFs) are known to be involved in smooth pursuit eye movements. It has been shown recently that stimulation of the smooth-pursuit area of the FEF [frontal pursuit area (FPA)] in monkey increases the pursuit response to unexpected changes in target motion during pursuit. In the current study, we applied transcranial magnetic stimulation (TMS) to the FPA and SEF in humans during sinusoidal pursuit to assess its effects on the pursuit response to predictable, rather than unexpected, changes in target motion. For the FPA, we found that TMS applied immediately before the target reversed direction increased eye velocity in the new direction, whereas TMS applied in mid-cycle, immediately before the target began to slow, decreased eye velocity. For the SEF, TMS applied at target reversal increased eye velocity in the new direction but had no effect on eye velocity when applied at mid-cycle. TMS of the control region (leg region of the somatosensory cortex) did not affect eye velocity at either point. Previous stimulation studies of FPA during pursuit have suggested that this region is involved in controlling the gain of the transformation of visual signals into pursuit motor commands. The current results suggest that the gain of the transformation of predictive signals into motor commands is also controlled by the FPA. The effect of stimulation of the SEF is distinct from that of the FPA and suggests that its role in sinusoidal pursuit is primarily at the target direction reversal.

Key words: smooth pursuit; eye movement; gain; supplementary eye field; frontal eye field; transcranial magnetic stimulation

\section{Introduction}

Smooth-pursuit eye movements are slow eye movements used to track a moving object. They are controlled by a network of cortical and subcortical brain regions (Leigh and Zee, 1999) that include the frontal eye fields (FEFs) and supplementary eye fields (SEFs).

The pursuit area of the FEF [frontal pursuit area (FPA)] in the macaque monkey is circumscribed to a small region in the fundus of the arcuate sulcus (MacAvoy et al., 1991). Microstimulation of this area evokes smooth pursuit but not saccades (Gottlieb et al., 1993). Microstimulation of the FPA increases the smooth pursuit response to target perturbations (Tanaka and Lisberger, 2001). Lesions to the FPA in monkeys and humans reduce pursuit eye velocity (Lynch, 1987; Keating, 1991; Morrow and Sharpe, 1995).

The SEF in the dorsomedial frontal cortex of the monkey has been implicated primarily in pursuit initiation and in direction reversals during periodic pursuit. Pursuit-related activity in the SEF is strongest immediately before pursuit initiation and at predictable target reversals (Heinen and Liu, 1997). Microstimulation of the SEF elicits smooth eye movements, increases smooth

Received July 6, 2005; revised Nov. 11, 2005; accepted Nov. 12, 2005.

This work was supported by operating grants from the Canadian Institutes of Health Research (T.P., G.B.P., and G.A.O.). D.G. was supported by National Science and Engineering Research Council of Canada Postgraduate Fellowships. M.-H.G. was supported by a grant from the Fyssen Foundation (Paris, France). G.B.P. and G.O'D. are William Dawson Scholars.

Correspondence should be addressed to Dr. Gillian A. O'Driscoll, Department of Psychology, McGill University, 1205 Dr. Penfield Avenue, Montreal, Quebec, Canada H3A 1B1. E-mail: gillian@hebb.psych.mcgill.ca. DOI:10.1523/JNEUROSCI.2789-05.2006

Copyright $\odot 2006$ Society for Neuroscience $\quad$ 0270-6474/06/260458-09\$15.00/0 eye velocity and acceleration during pursuit initiation, and increases anticipatory pursuit (Tian and Lynch, 1996; Missal and Heinen, 2001, 2004). Lesions to the SEF have been reported to increase the latency to reverse direction during periodic pursuit (Heide et al., 1996).

These data suggest that oculomotor regions may make different contributions to different portions of the pursuit cycle. Here, we investigated the contributions of the FPA and SEF to sinusoidal tracking at mid-cycle and at the target direction reversal.

Transcranial magnetic stimulation (TMS) uses a magnetic field to carry an electrical current through the skull to the cortex. The current temporarily affects neural activity in a focal region under the coil (Jahanshahi and Rothwell, 2000). TMS can activate the cortex to enhance normal behavior or disrupt cortical processing to interfere with ongoing behavior (Cracco et al., 1999; Jahanshahi and Rothwell, 2000). Enhancing effects of TMS include improved performance on visual tasks with stimulation of the FEF (Grosbras and Paus, 2002, 2003). Disruptive effects of TMS include suppression of visual perception with stimulation of the visual cortex and delayed reaction times with stimulation of the motor cortex (Cracco et al., 1999; Jahanshahi and Rothwell, 2000).

The effect of single-pulse TMS on oculomotor regions has been investigated primarily in the context of saccade tasks. TMS of oculomotor regions does not evoke saccades (Muri et al., 1991; Wessel and Kompf, 1991) but increases the latencies of visually guided and voluntary saccades (Priori et al., 1993; Terao et al., 1998) and increases saccade-sequence errors (Muri et al., 1995). 
Only one study has applied TMS during pursuit (Ohtsuka and Enoki, 1998). TMS of the cerebellum increased pursuit acceleration ipsilateral to the stimulation. Thus, TMS can modulate smooth pursuit performance. Here, we describe experiments in which we apply TMS to the FPA, SEF, and two control regions during sinusoidal pursuit, first at mid-cycle and then at the target turnaround. TMS of the FPA modulated eye velocity when applied at both places, whereas TMS of the SEF modulated eye velocity only at the target direction reversal.

\section{Materials and Methods Experiment 1}

\section{Subjects}

Twenty-eight right-handed subjects ( 7 males and 21 females) with an average age of 22.1 years $(S D, \pm 9.5)$ were screened in the laboratory using the same pursuit task that was used in the scanner. Of these, nine subjects with pursuit gain $>0.95$ and who made few saccades participated in the functional magnetic resonance imaging (fMRI) and TMS sessions. These subjects (average age, 23.8 years; SD, \pm 3.3 ) reported no history of neurological disorders and did not meet criteria for any psychiatric or substance abuse disorders as assessed by the Diagnostic Interview Schedule (Robins et al., 1981). All subjects provided written informed consent and were compensated for their participation. The experimental protocol was approved by the Research Ethics Board of the Montreal Neurological Institute/Hospital (MNI/H).

\section{MRI}

Structural. Anatomical MRIs were acquired for all nine subjects participating in the TMS session. For the first six subjects, fMRI data were acquired to confirm that the task reliably activated the predicted anatomical areas. Once this was established, the average coordinates of activation were used to localize the site of TMS on the structural MRIs of the remaining subjects. Three-dimensional, high-resolution structural MRIs were acquired on a 1.5 tesla Vision or Magnetom SonataVision scanner (Siemens Medical Systems, Erlangen, Germany) using a spoiled gradient-recalled echo (GRE) pulse sequence (Vision: repetition time (TR), $22 \mathrm{~ms}$; echo time (TE), $10 \mathrm{~ms}$; flip angle, $30^{\circ}$; Magnetom SonataVision: TR, $22 \mathrm{~ms}$; TE, $9.2 \mathrm{~ms}$; flip angle, $30^{\circ}$ ). This yielded T1-weighted slices with $1 \mathrm{~mm}$ thickness and an effective in-plane resolution of $1 \times 1$ $\mathrm{mm}$ [field of view (FOV), $256 \mathrm{~mm}$; matrix, $256 \times 256$ ].

Functional. T2*-weighted functional images sensitive to bloodoxygen-level-dependent (BOLD) contrast were acquired using a singleshot GRE echo-planar imaging sequence (TR, $0.8 \mathrm{~ms}$; TE, $50 \mathrm{~ms}$; flip angle, $90^{\circ}$ ). Sixteen 6-mm-thick axial-oblique slices aligned with the anteroposterior commissure were selected from sagittal scout images acquired about the midline. In-plane resolution for these images was $5 \times 5$ $\mathrm{mm}$ (FOV, $320 \mathrm{~mm}$; matrix, $64 \times 64$ ). Localized shimming of the slices was used to maximize the magnetic field homogeneity. Each subject's head was immobilized using a vacuum bag custom-molded to each subject's head.

Subjects performed two oculomotor tasks, smooth pursuit and fixation. In both tasks, the target was a red square subtending $0.5 \times 0.5^{\circ}$ of visual angle presented on a black background. (1) In smooth pursuit, subjects tracked the target moving horizontally across $20^{\circ}$ of visual angle at a frequency of $0.4 \mathrm{~Hz}$ with a sinusoidal velocity profile (peak target velocity, $25.1 \%$ s). (2) During fixation, the target remained stationary in the center of the screen. Subjects were instructed to maintain their gaze on the target for the duration of the task.

Smooth pursuit and fixation were alternated every $30 \mathrm{~s}$ for a period of $6 \mathrm{~min}$ to form a "run" that each subject performed three times. The order of tasks was counterbalanced within and between subjects. For each of the three runs, 120 scans of $3 \mathrm{~s}$ duration each were acquired, yielding a total of 360 scans for each subject.

The subjects viewed the stimuli via a MultiSync MT1030+ (NEC Technologies, Itasca, IL) active-matrix projector running in $1024 \times 768$ mode at $60 \mathrm{~Hz}$ with a light output of 1100 ANSI lumens and a contrast ratio of 300:1. The projector was connected to a personal computer supporting EyeLink software (SR Research, Mississauga, Ontario, Canada) via a DB15 VGA cable. A mirror system positioned above the head coil allowed the subjects to view a screen positioned behind them in the bore of the magnet.

\section{TMS}

All TMS was administered with a Magstim 200 (Whitland, Wales, UK) rapid magnetic stimulator delivered through a figure- 8 coil.

Positioning of the coil and TMS setup. The TMS coil was positioned over each region of interest (ROI) (see below) with the aid of frameless stereotaxy software (Brainsight; Rogue Research, Montréal, Quebec, Canada), an optical tracking system (Polaris; Northern Digital, Waterloo, Ontario, Canada), and each subject's native MRI. Each subject's head position was measured using the Polaris tracking system to identify the location of scalp landmarks (nasion, nose-tip, intra-trageal notch of the left and right ear) visible on each subject's MRI. Once the subject's head and MRI were coregistered, each site of stimulation was localized. The location of a digitizing pen about the subject's head was measured using the Polaris system and transferred in real time to the computer loaded with the Brainsight software. The digitizing pen was moved to the individual coordinates of peak activity for the relevant region for subjects who participated in the fMRI session and to the coordinates of the group peak for the remaining subjects. Locations were marked on the subject's scalp with a grease pencil.

The stimuli were presented on a 17 -inch NEC Technologies monitor running at $135 \mathrm{~Hz}$. Eye movements were monitored during the TMS session using a $250 \mathrm{~Hz}$ infrared pupil tracker (Eyelink; SR Research). A three-target calibration and validation was performed across $28^{\circ}$ of visual angle before the onset of the tasks. The average fixation error on validation was $<0.5^{\circ}$, which is consistent with the expected variability in fixation. The eye-camera was mounted on a horizontal bar in front of the subject, which also held a mechanical arm, chin rest, and bite bar. The mechanical arm, which has freedom of motion in all three planes, was used to hold the TMS coil in place. The subject's head was held in place by the chin rest and a personalized bite bar made of dental plastic. This system allowed subjects to take a break between testing blocks and to return to the same position when testing was resumed. During the application of TMS pulses, the clicks associated with the stimulation were attenuated with earplugs.

Establishing the motor threshold. The stimulation intensity of the TMS coil was determined for each subject using the individual motor threshold (Grosbras and Paus, 2002). Motor twitches were observed from the right first dorsal interosseous muscle while the stimulation intensity was increased until motor twitches were elicited in 5 of 10 trials. This was considered the subject's motor threshold, and the stimulation intensity was set at $10 \%$ above this threshold.

TMS during smooth pursuit. Subjects pursued a target moving horizontally at $0.4 \mathrm{~Hz}$ across $20^{\circ}$ of visual angle for $120 \mathrm{~s}$. Twenty single pulses of TMS were pseudorandomly applied throughout the $120 \mathrm{~s}$, with a minimum separation of $5 \mathrm{~s}$, with an equal number of pulses delivered during pursuit to the left and to the right. TMS pulses were always applied at the same point within a half-cycle (i.e., the point of peak target velocity). For each brain region (defined below), this procedure was performed twice, with a break between recording sessions. In total, 40 TMS pulses were acquired for each region, 20 while subjects pursued to the left and 20 while subjects pursued to the right. TMS pulses were synchronized with stimulus movement using the eye-tracking software. The order in which regions were stimulated was counterbalanced across subjects.

\section{Analysis}

Functional imaging data. Using the third scan of each run as the reference, the functional data were motion corrected by coregistering all time points using a local routine developed at the MNI. Functional data from each run was then subjected to a low-pass filter with a $6 \mathrm{~mm}$ full-width half-maximum (FWHM) Gaussian kernel.

Significant increases in the BOLD signal for smooth pursuit minus fixation for each run were calculated using in-house software (Worsley et al., 2000). The analysis was based on a general linear model with correlated errors. The hemodynamic response was first modeled as the differ- 
Table 1. Individual FPA and SEF coordinates for pursuit $>$ fixation

\begin{tabular}{|c|c|c|c|c|c|c|c|c|c|c|c|c|}
\hline \multirow[b]{2}{*}{ Subject } & \multicolumn{4}{|l|}{ IFPA } & \multicolumn{4}{|c|}{ rFPA } & \multicolumn{4}{|l|}{ SEF } \\
\hline & $x$ & $y$ & $z$ & $t$ score $^{a}$ & $x$ & $y$ & $Z$ & $t$ score & $x$ & $y$ & $z$ & $t$ score \\
\hline S1 & -34 & -10 & 44 & 4.79 & 40 & 3 & 55 & 6.71 & -7 & -4 & 56 & 3.89 \\
\hline $\mathrm{S} 2$ & -35 & -3 & 43 & 2.93 & 31 & -1 & 41 & 4.76 & -7 & -3 & 70 & 3.00 \\
\hline S3 & -34 & -12 & 53 & 4.89 & 38 & -8 & 58 & 8.71 & -8 & -6 & 53 & 6.08 \\
\hline S4 & -47 & -10 & 41 & 6.49 & 33 & -16 & 49 & 5.47 & 0 & -11 & 47 & 4.79 \\
\hline S5 & -26 & -16 & 56 & 6.45 & 24 & -15 & 51 & 7.79 & 2 & -4 & 55 & 4.38 \\
\hline S6 & -39 & -2 & 50 & 6.69 & 42 & -4 & 56 & 7.49 & 7 & -5 & 64 & 5.43 \\
\hline Mean & -36 & -9 & 48 & 5.86 & 35 & -7 & 52 & 6.82 & -2 & -6 & 58 & 4.60 \\
\hline SE & 3 & 2 & 2 & 0.38 & 3 & 3 & 2 & 0.60 & 2 & 1 & 3 & 0.45 \\
\hline
\end{tabular}

${ }^{a}$ All t scores $\geq 3.00$ are significant $(p<0.05)$.

ence of two gamma-density response functions with a mean lag of $6 \mathrm{~s}$ and a SD of $3 \mathrm{~s}$. The autocorrelation parameter was regularized with a $15 \mathrm{~mm}$ FWHM Gaussian filter, and drift artifact was removed up to the third polynomial. The first two scans of each run were also excluded from the analysis because the scanner has not reached "steady-state" transverse magnetization. Significant increases in activity were then modeled as an autoregressive process of degree- 1 for each of the three runs (Bullmore et al., 1996). Each subject's three runs were than averaged using a randomeffects analysis. The resulting individual functional maps were then resampled at a higher resolution $(2 \times 2 \times 2 \mathrm{~mm})$. The individual functional maps were then coregistered with each subject's native anatomical MRI. Images were thresholded at a $t$ value of 3.00 (the minimum given by a Bonferroni's correction and random-field theory; $p<0.05$ ) (Worsley et al., 1996) based on the search volume for the $\operatorname{FEF}\left(330 \mathrm{~mm}^{3}\right)$, centered at the junction of the precentral and superior frontal sulci (Paus, 1996), which we have used in a previous study (O'Driscoll et al., 1999). The same size search volume (centered on a different location) was used to identify activation in the SEF, and results were thresholded to the same $t$ value.

There were three primary ROIs: the left FPA (IFPA), right FPA (rFPA), and SEF. As a control region, we selected an area implicated in arm movements, thought to correspond to the human homolog of the anterior intraparietal area (AIP) in macaque (for review, see Culham and Kanwisher, 2001; Grefkes and Fink, 2005). This area is anterior to the parietal regions generally implicated in eye movements. Because of the current uncertainty of the homology between the AIP and this region in humans, we refer to this region as the rostral parietal region (rosPAR). The Talairach coordinates for stimulation were taken from a study that compared reaching during fixation with fixation alone (Kawashima et al., 1996) ( $x=30, y=-35, z=50)$. The coordinates of the other regions were determined as the peak activity in the search volume of that region.

TMS. We used previous studies to determine the segment of pursuit that would be analyzed for an effect of TMS. A recent study demonstrated that the latency of the behavioral response to electrical stimulation of the FPA during pursuit in non-human primates was $\sim 22 \mathrm{~ms}$ (Tanaka and Lisberger, 2002). Behavioral effects of single-pulse TMS effects in humans can persist up to $150 \mathrm{~ms}$ after stimulation (Jahanshahi and Rothwell, 2000). We therefore used an analysis window of 25-125 ms after stimulation.

Mean eye velocity was calculated in each analysis window for cycles when TMS was applied and cycles when it was not, separately for each direction (i.e., leftward pursuit, with TMS; leftward pursuit, without TMS; rightward pursuit, with TMS; rightward pursuit, without TMS). Semi-automated software (SR Research) was used to analyze the pursuit traces. Any window that contained a blink or a saccade (eye acceleration, $>4000 \% \mathrm{~s}^{2}$; increase in eye velocity, $>22 \%$ ) (Gagnon et al., 2002) was excluded from the pursuit analysis.

For each region (IFPA, rFPA, SEF, and rosPAR), we tested for differences in smooth eye velocity as a result of TMS using a two-way, withinsubjects ANOVA with direction of pursuit (left vs right) as one factor and TMS (applied or not) as the other factor. We hypothesized that TMS of the FPA would increase eye velocity because stimulation of FPA in the monkey increases eye velocity (Gottlieb et al., 1993). We did not hypothesize an effect of TMS of the SEF or rosPAR. We analyzed the number of analysis windows that were excluded for saccades to determine whether
TMS, which does not evoke saccades during fixation (Muri et al., 1991; Wessel and Kompf, 1991; Zangemeister et al., 1995), might evoke saccades in the context of pursuit.

\section{Experiment 2}

In experiment 1, in which TMS was applied at peak target velocity, we found a significant effect of FPA stimulation but no effect of SEF stimulation. However, the SEF is significantly activated during pursuit (Berman et al., 1999; Petit and Haxby, 1999; O’Driscoll et al., 2000). Singleunit studies have reported that SEF activity is highest in sinusoidal pursuit immediately preceding a predictable change in target direction (Heinen and Liu, 1997). A study of patients with lesions of the SEF found a delay in reversing direction during sinusoidal pursuit (Heide et al., 1996). We therefore hypothesized that TMS of the SEF might affect pursuit if applied immediately before the target direction reversal, although it had no effect at peak target velocity. Experiment 2 was designed to test this hypothesis.

\section{Subjects}

Seven right-handed subjects with an average age of 23.7 years (SD \pm 3.7$)$ participated in the second experiment. Six of these subjects had participated in the first experiment. One new subject was recruited and went through the identical psychophysical and medical screening as the rest of the subjects. The subject also provided a structural MRI with the same parameters as described above. The Research Ethics Board of the MNI/H approved the experimental protocol. All subjects provided written informed consent and were compensated for their time.

\section{TMS}

The TMS procedure was the same as in experiment 1 , with the exception that the TMS pulse was applied $75 \mathrm{~ms}$ before the target turnaround rather than at the point of peak target velocity. We selected $75 \mathrm{~ms}$ before the turnaround because the activity of pursuit-related neurons in the SEF peaks before the eye reverses direction (Heinen and Liu, 1997) and facilitation of pursuit initiation occurs with stimulation of the SEF beginning $75 \mathrm{~ms}$ before target motion onset (Missal and Heinen, 2001).

In each half cycle, direction reversal was defined as the first point after the eye had reached its extreme eccentricity that consecutive position samples indicated movement back to center. For each ROI (lFPA, rFPA, SEF, and rosPAR), a two-way, within-subjects ANOVA was run with direction of smooth pursuit (left vs right) as one factor and TMS (applied and not applied) as the other factor. Based on a previous study (Heide et al., 1996), we also examined the effect of TMS on the latency of the eye to reverse direction. Finally, we analyzed whether TMS at the target reversal elicited saccades: we performed a TMS by direction ANOVA for all ROIs using number of analysis windows excluded because of saccades as the dependent variable.

\section{Experiment 3}

In experiment 2, TMS applied to the FPA and SEF increased smooth eye velocity in both directions, but TMS applied to the rosPAR (the control region) also affected eye velocity when pursuit after the turnaround was contralateral to the stimulation (i.e., to the left). To clarify whether the effect of TMS at the turnaround is mediated by stimulation of specific task-relevant brain regions or by nonspecific alerting factors, we ran a 


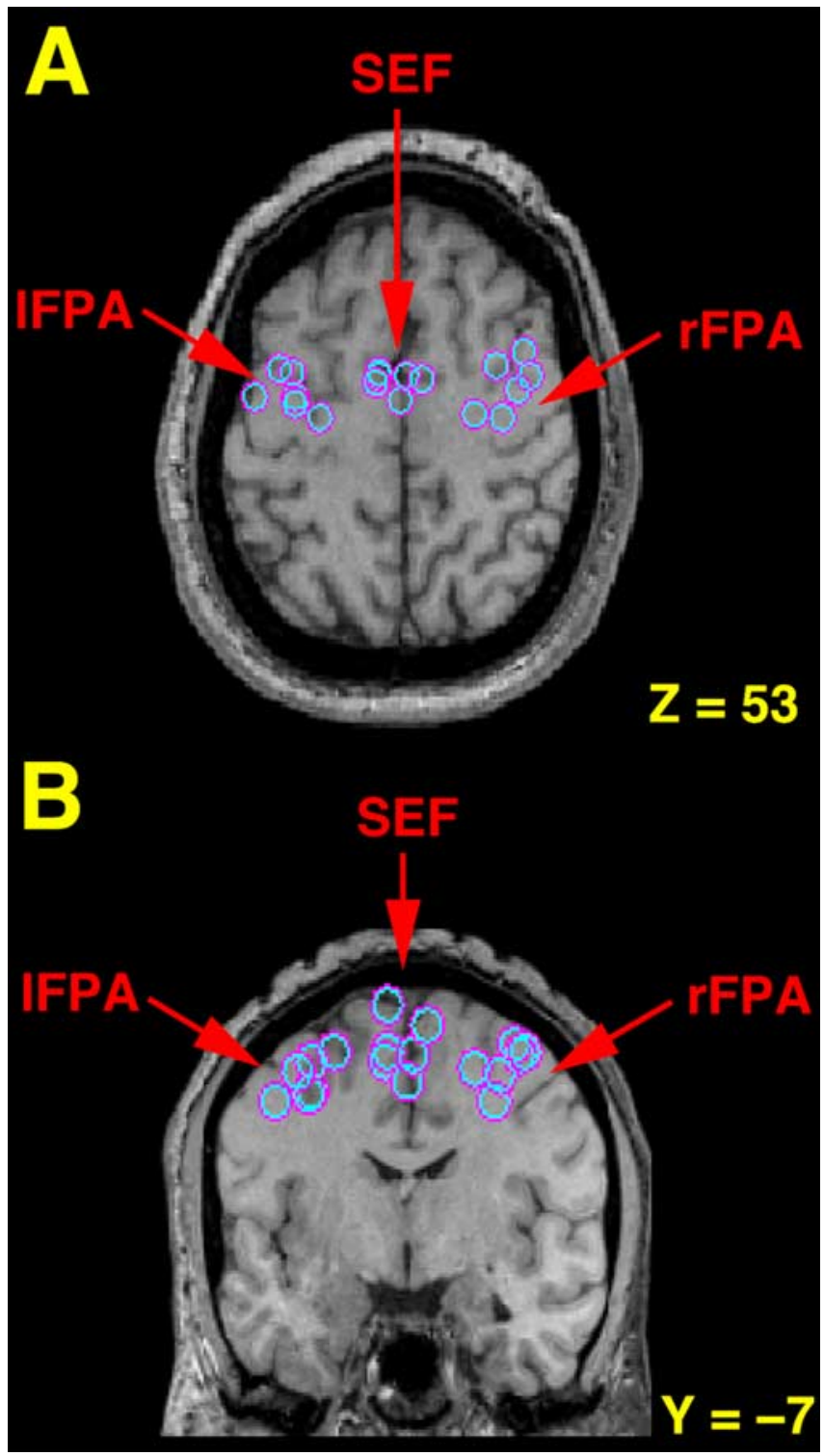

Figure 1. Transverse $(\boldsymbol{A})$ and coronal $(\boldsymbol{B})$ sections illustrating the location for each subject $(n=6)$ of the IFPA, rFPA, and SEF as determined by fMRI comparing smooth pursuit to fixation. The left hemisphere is shown on the left side of each image. The locations are superimposed on a single subject's MRI, which has been transformed into standard Talairach space. For illustrative purposes, the horizontal slice $(z=53)$ was selected based on the average $z$ coordinates of activation for the FPA and SEF as determined by fMRI. The coronal section $(y=-7)$ was selected based on the average $y$ coordinates of activation for the FPA and SEF. Note that the greatest activity in the FPA was consistently found at or near the junction of the superior frontal and precentral sulci $(\boldsymbol{A})$ and that increased activity in the SEF was regularly found in the medial wall near the paracentral sulcus $(\boldsymbol{B})$, both established anatomical landmarks for these regions in humans (Paus, 1996; Grosbras et al., 1999).

third TMS experiment in which the control region was an area with no known projections to oculomotor regions [the leg region of the primary somatosensory cortex (leg-S1)]. If stimulation of the new control region produced an effect on pursuit, it could be considered to be the result of nonspecific factors. However, if stimulation of the new control region did not, but stimulation of oculomotor regions did, the effect of TMS could be attributed to action on the oculomotor circuit.

\section{Subjects}

Six subjects from experiment 2 were available and returned for testing in this experiment. The protocol was approved by the Research Ethics
Table 2. Experiment 1: main effect of TMS when applied at peak target velocity

\begin{tabular}{|c|c|c|c|}
\hline \multirow[b]{2}{*}{ Region } & \multicolumn{2}{|c|}{ Velocity $\left({ }^{\circ} / \mathrm{s}\right)$} & \multirow{2}{*}{$\begin{array}{l}\text { Average percentage } \\
\text { decrease }\end{array}$} \\
\hline & No TMS & TMS & \\
\hline IFPA & $24.6(0.4)$ & $23.4(0.4)$ & $4.8(1.0)^{* *}$ \\
\hline rFPA & $24.2(0.3)$ & $23.1(0.6)$ & $4.7(1.9)^{*}$ \\
\hline SEF & $24.2(0.3)$ & $23.7(0.5)$ & $1.9(1.7)$ \\
\hline rosPAR & $24.5(0.4)$ & $24.0(0.4)$ & $1.9(0.9)$ \\
\hline
\end{tabular}

Data are the mean (SE) of pursuit eye velocity. ${ }^{*} p<0.05 ;{ }^{* *} p<0.01$.
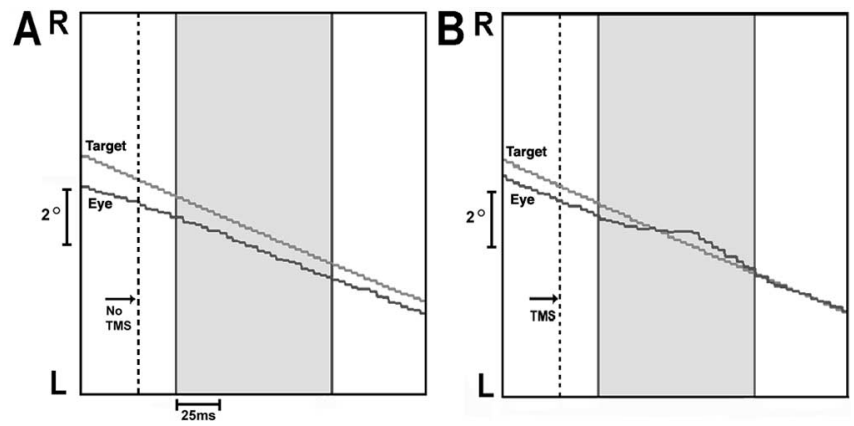

Figure 2. Experiment 1. The effect of TMS on eye velocity when applied at peak target velocity in one subject. The $x$-axis is time (milliseconds), and the $y$-axis is position. The panels depict pursuit at midcycle of a target moving horizontally at $0.4 \mathrm{~Hz}$ with a sinusoidal velocity profile across $20^{\circ}$ of visual angle. The dotted line indicates the point of peak target velocity, the moment that TMS was applied in $\boldsymbol{B}$. The gray portion of each panel illustrates the $100 \mathrm{~ms}$ analysis window that begins $25 \mathrm{~ms}$ after peak target velocity. $\ln \boldsymbol{A}$ (No TMS), eye velocity is close to target velocity (average eye velocity in window, $23.0^{\circ} / \mathrm{s}$; average target velocity, $24.4^{\circ} / \mathrm{s}$ ). In $\boldsymbol{B}$ (TMS of IFPA), eye velocity in the window is reduced (average eye velocity, $20.0 \%$; average target velocity, $\left.24.4^{\circ} / \mathrm{s}\right) . \mathrm{R}$, Right; L, left.

Board of the MNI/H. Subjects provided written informed consent and were compensated for their time and effort.

\section{TMS}

TMS was applied in two separate testing blocks, one with TMS applied at peak target velocity, and the other with stimulation applied at the target direction reversal. The order of blocks was counterbalanced across subjects. Two ROIs were tested in each block, the leg-S1 and the IFPA, which had previously yielded significant effects of TMS at both points in the cycle. The Talairach coordinates of leg-S1 were calculated from previous neuroimaging studies (Fink et al., 1997; Debaere et al., 2001; Stippich et al., 2002). Coordinates across these studies were highly similar and separated by a maximum vector distance of only $13 \mathrm{~mm}$. The coordinates used (average of the three studies: $x=-7, y=-41, z=63$ ) were transformed into each subject's native space. The analysis procedure was identical to that performed in experiments 1 and 2 .

\section{Results \\ Experiment 1 \\ $f M R I$}

Greater activity in the IFPA, rFPA, and SEF was observed when smooth pursuit was compared with fixation in all subjects, replicating the results of previous studies (Berman et al., 1999; Petit and Haxby, 1999; O'Driscoll et al., 2000) (Table 1). The average coordinates of the FPA were as follows: $x= \pm 36, y=-8$, and $z=$ 50. This location is consistent with localization of this region in previous studies (Paus, 1996; Petit and Haxby, 1999; O'Driscoll et al., 2000) (Fig. 1). Increased activity in the SEF was found to be located in medial area 6 in the region of the paracentral sulcus, identified previously as the neuroanatomical landmark identifying the location of the SEF (Grosbras et al., 1999) (Fig. 1). 
TMS

Eye velocity analysis. There was a significant main effect of TMS of the IFPA $\left(F_{(1,8)}=\right.$ 19.26; $p<0.002)$ and $\operatorname{rFPA}\left(F_{(1,7)}=6.10\right.$; $p<0.04)$ on smooth eye velocity. TMS of the $\mathrm{SEF}$ and rosPAR did not significantly affect smooth eye velocity $(p>0.05)$ (Table 2$)$. There was no effect of pursuit direction (left or right) on smooth eye velocity and no interaction between direction and TMS (all $p>$ 0.05). Thus, post hoc analyses were done with smooth eye velocity averaged across directions. With TMS of the IFPA, eye velocity $( \pm \mathrm{SE})$ decreased from $24.6 \%( \pm 0.4)$ to $23.4 \%$ s $( \pm 0.4)$, with an average withinsubject reduction of $4.8 \%$ ( \pm 1.0$)$. With TMS of the rFPA, the average eye velocity decreased from $24.2 \%( \pm 0.3)$ to $23.1 \%$ $( \pm 0.6)$, with an average within-subject decrease of $4.7 \%( \pm 1.9)$ (Table 2$)$. The effect of TMS on pursuit eye velocity in one subject is illustrated in Figure 2. Eye velocity decreased after stimulation of the IFPA in eight of nine subjects and decreased after stimulation of the rFPA in six of eight subjects (one subject blinked each time TMS was applied to the rFPA, and thus the effect on eye velocity could not be examined) (Fig. 3).

Saccade analysis. We analyzed the proportion of trials in which saccades occurred during the analysis window for TMS and noTMS cycles. There was no effect of TMS and no interaction between TMS and direction for any ROI (all $p>0.05$ ).

\section{Experiment 2}

MRI

The TMS coil was placed at the same locations as in experiment 1 for six subjects (Table 1, S1-S6). To determine coil placement for the new subject, the peak coordinates of activation from the first six subjects for each ROI were transformed into the new subject's native space using her structural MRI.

\section{TMS}

Eye velocity analysis. An example of the effect of TMS on eye velocity in a single subject is shown in Figure 4. The effect of TMS on each subject's smooth eye velocity for each ROI is illustrated in Figure 5.

TMS applied to the FPA and to the SEF significantly increased eye velocity in the new pursuit direction $\left(\right.$ IFPA: $F_{(1,6)}=10.50, p<$ 0.018; rFPA: $F_{(1,6)}=6.89, p<0.039$; SEF: $F_{(1,6)}=18.06, p<$ 0.005 ) (Table 3 ). With TMS of the IFPA, eye velocity ( \pm SE) increased from $4.1 \% \mathrm{~s}( \pm 0.3)$ to $5.1 \% \mathrm{~s}( \pm 0.5)$, with an average within-subject increase of $24.5 \%( \pm 6.4)$. TMS of the rFPA increased eye velocity from $4.0 \% \mathrm{~s}( \pm 0.2)$ to $5.8 \%$ s ( \pm 0.8$)$, an average within-subject increase of $43.7 \%( \pm 15.0)$. TMS of the SEF increased eye velocity from $4.3 \% \mathrm{~s}( \pm 0.2)$ to $5.5 \% \mathrm{~s}( \pm 0.3)$, an average within-subject increase of $29.3 \%$ ( \pm 7.0$)$. There was no main effect of direction and no interaction of TMS and direction. In Table 3, we report the average velocities with and without TMS averaging across direction, as well as the average percentage change in velocity from the no-TMS condition to the TMS condition. There was a significant interaction between TMS and direction in the rosPAR $\left(F_{(1,6)}=18.15 ; p<.005\right)$. TMS of the rosPAR (which was in the right hemisphere) increased eye veloc-
A
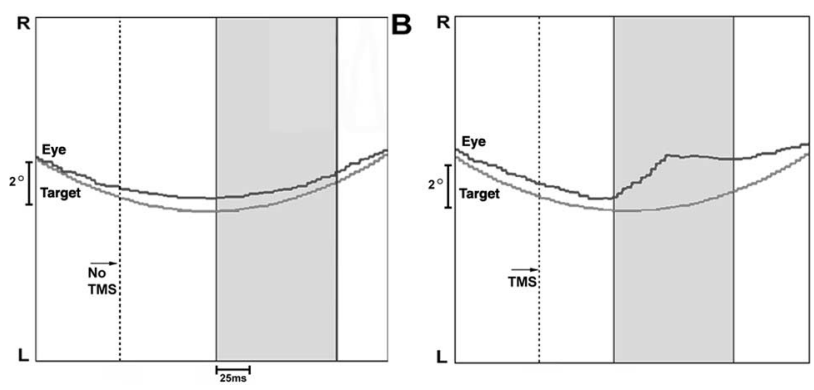

Figure 4. Experiment 2. An example of the effect of TMS on eye velocity when applied at the target direction reversal in one subject. The $x$-axis is time, and the $y$-axis is position. The panels depict pursuit at the target direction reversal; the target is moving horizontally at $0.4 \mathrm{~Hz}$ with a sinusoidal velocity profile across $20^{\circ}$ of visual angle. The dotted line is placed $75 \mathrm{~ms}$ before the target direction reversal, the moment at which TMS was applied in $\boldsymbol{B}$. The gray portion of each panel illustrates the $100 \mathrm{~ms}$ analysis window that began the moment the eye reversed direction (see Materials and Methods). $A$, No TMS: the average eye velocity in this analysis window was $2.7 \%$ (average target velocity, $3.5 \%$ ). $B$, TMS of the IFPA: the average eye velocity in this analysis window was $4.8^{\circ} / \mathrm{s}$ (average target velocity, $3.2^{\circ} / \mathrm{s}$ ). R, Right; L, left.

ity $( \pm \mathrm{SE}$ ) when pursuit after the turnaround was to the left (no TMS, $4.0 \% \pm 0.2$; TMS, $5.7 \% \pm 0.6$ ) with an average increase of $38.1 \% \pm 0.2$, but did not affect eye velocity when pursuit after the turnaround was to the right (no TMS, $4.3 \% \pm 0.3 ; \mathrm{TMS}, 4.2^{\circ} \%$ 土 0.3 ; percentage change, $1.2 \% \pm 0.1)$.

Latency analysis. There was no effect of TMS for any region on mean latency (in ms \pm SE) for the eye to reverse direction (IFPA: no TMS, $2.8 \pm 5.0$; TMS, $6.9 \pm 8.5$; rFPA: no TMS, $0.9 \pm 2.5$; TMS, $1.6 \pm 4.9$; SEF: no TMS, $3.8 \pm 3.2$; TMS, $8.6 \pm 5.4$; rosPAR: no TMS, $0.3 \pm 3.4$; TMS, $0.9 \pm 7.1$ ), and TMS did not interact with pursuit direction (all $p>0.05$ ) (Fig. 6).

Saccade analysis. The number of saccades at the turnaround was not increased by TMS of any ROI: there was no main effect of TMS and no interaction of TMS with direction for any region (all $p>0.05)$. 

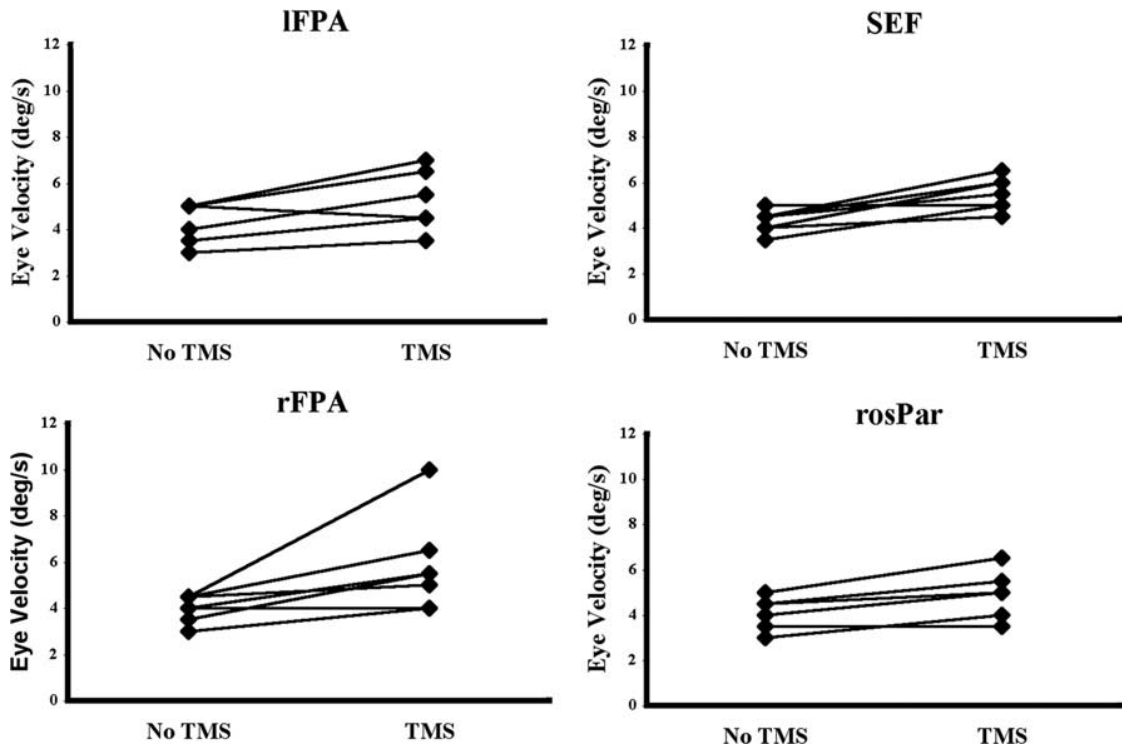

Figure 5. Experiment 2. Effect of TMS on eye velocity when applied at the target direction reversal. Each individual's eye velocity with and without TMS is connected by a straight line. TMS of each ROI significantly increased eye velocity.

Table 3. Main effect of TMS when applied at the target turnaround

\begin{tabular}{llll}
\hline \multirow{2}{*}{ Region } & \multicolumn{2}{l}{ Velocity $\left({ }^{\circ} / \mathrm{s}\right)$} & \\
\cline { 2 - 3 } & No TMS & TMS & \\
\hline IFPA & $4.1(0.3)$ & $5.1(0.5)$ & $24.5(6.4)^{*}$ \\
rFPA & $4.0(0.2)$ & $5.8(0.8)$ & $43.7(15.0)^{*}$ \\
SEF & $4.3(0.2)$ & $5.5(0.3)$ & $29.3(7.0)^{* *}$ \\
rosPAR & $4.1(0.3)$ & $4.9(0.4)$ & $19.0(4.5)^{* *}$ \\
\hline
\end{tabular}

Data are the mean (SE) of pursuit eye velocity. ${ }^{*} p<0.05 ;{ }^{* *} p<0.01$.

\section{Experiment 3}

TMS applied to the IFPA at peak target velocity significantly decreased eye velocity $\left(F_{(1,5)}=5.93 ; p<0.002\right)$, but TMS of leg-S1 at the same point did not (Table 4, Fig. 7). TMS applied to the IFPA at the target direction reversal increased eye velocity $\left(F_{(1,5)}\right.$ $=6.79 ; p<0.048$ ), whereas TMS of leg-S1 did not (Table 4, Fig. $7)$. There were no interactions between TMS and direction ( $p>$ 0.05).

Thus, TMS of the IFPA was again associated with robust effects on eye velocity at both peak target velocity and the target turnaround, but stimulation of the leg-S1 was not. This suggests that the effects observed in experiment 2 were not attributable to nonspecific effects of TMS, such as "alerting" related to the click, but were attributable to action on the oculomotor circuit.

\section{Discussion}

The main finding of the current study was that TMS applied to the FPA immediately before the target direction reversal increased the eye's initial velocity in the new direction, whereas TMS applied to the FPA in the middle of the trajectory of the target (i.e., the moment before the target began to slow down) decreased eye velocity. In contrast, TMS applied to the SEF at the target turnaround increased eye velocity but had no effect when applied in the middle of the target trajectory. Stimulation of a non-oculomotor control region (leg-S1) did not result in significant changes in eye velocity. Previous studies have indicated that stimulation of the FPA in monkeys increases the gain of the transformation of visual signals to motor commands (Tanaka and Lisberger, 2001). The current findings suggest that the FPA also increases the gain of the transformation of predictive signals that are thought to drive high-gain, sinusoidal pursuit. Below we discuss the results in relation to previous studies of the FPA and SEF and consider possible mechanisms of the effects observed.

\section{FPA}

Pursuit theorists have generally posited the existence of a "pursuit switch" to explain the increased sensitivity to velocity signals during pursuit compared with fixation (Robinson, 1965; Grasse and Lisberger, 1992; Keating and Pierre, 1996). This switch is thought to increase the gain of the transformation of visual signals to motor output (Tanaka and Lisberger, 2001). The location of this switch or gain controller in the brain has been proposed to be the FPA (Tanaka and Lisberger, 2001), because lesions to the FPA produce behavioral effects that could be consistent with "jamming-open" of the switch (Lynch, 1987; Keating, 1991). Specifically, FPA lesions abolish anticipatory pursuit (Keating, 1991; MacAvoy et al., 1991) in which the intent to pursue is thought to close the pursuit switch (Keating and Pierre, 1996). Furthermore, lesions of the FPA reduce the gain of pursuit initiation and maintenance (Keating, 1991, 1993; Morrow and Sharpe, 1995; Lekwuwa and Barnes, 1996). Microstimulation of the FPA in non-human primates increases eye velocity during pursuit of a constant velocity target (Gottlieb et al., 1993), which could reflect either the injection of a velocity signal or the turning-up of the gain controller. However, FPA stimulation produces an omnidirectional enhancement in the changes in eye velocity that occur in response to sudden changes in target movement (Tanaka and Lisberger, 2001), which is consistent with an effect on a gain controller.

For predictable, periodic targets, smooth pursuit generally has zero phase lag and near-unity gain (Stark et al., 1962; Yasui and Young, 1984; van den Berg, 1988), which are thought to reflect the use of "anticipatory," "predictive," or "cognitive" signals to track the target, in addition to retinal and eye velocity signals (Keating, 1991, 1993; MacAvoy et al., 1991). We believe the changes in eye velocity in the current study likely reflect a turning up of the gain of the predictive signals that drive sinusoidal pursuit. It has been shown previously that the latency of the effect of stimulation during pursuit of a constant velocity (predictable) target is $\sim 22 \mathrm{~ms}$ (Tanaka and Lisberger, 2001). The latency to affect pursuit responses to an unpredictable change in target motion was longer, with the maximum response occurring in a window 100-150 ms after the change. This delay is consistent with transmission delays from the retina to the cortex and brainstem (Lisberger and Westbrook, 1985). In the current study, the pursuit response was enhanced to changes in target motion that were unlikely to have had time to travel from the retina to the brainstem. Specifically, the increases in eye velocity at the target turnaround was in an analysis window that began the moment the eye reversed direction, on average $3 \mathrm{~ms}$ after the target reversed direction. Similarly, with TMS at peak target velocity, the decrease in eye velocity was observed in an analysis window that began only $25 \mathrm{~ms}$ after the target began to slow. Thus, we believe the effects observed here probably reflect a turning up of the gain of the predictive signals driving pursuit. Such an interpretation is consistent with models of sinusoidal pursuit that posit control 
mechanisms that are predominantly "internal" (i.e., based on predictions derived from previous experience with the target motion) (Dallos and Jones, 1963; van den Berg, 1988), rather than purely retinal.

An alternative hypothesis is that the differing effects on eye speed of FPA stimulation at the target turnaround and at peak target velocity are related to the level of activity in the FPA at the time of stimulation. In skeletomotor research, TMS of the hand area of the motor cortex produces muscle twitches when stimulation is applied if the hand is at rest. However, when the hand is clenched, stimulation of the same region induces a silent period in the EMG (for review, see Cracco et al., 1999). The implication is that TMS effects may be different depending on the current rate of neural firing in the region being stimulated. The firing rate in the FPA in monkeys is positively correlated with eye speed (Gottlieb et al., 1994). Thus, by analogy, it is possible that TMS of the FPA at the target turnaround (when average eye velocity is $<5 \%$ s) increased eye speed because the neural firing rate at that time was low; in contrast, TMS of the FPA at peak target velocity (when eye speed was $>20 \%$ ) may have decreased eye speed because the rate of neural firing was already high. Such an alternative explanation is difficult to rule out because the effect of TMS on neural systems is not well understood.

\section{SEF}

The SEF is well placed to play a role in smooth pursuit (Heinen and Liu, 1997). In monkeys, neurons in the SEF have been shown to fire during pursuit (Schall, 1991; Heinen, 1995; Heinen and Liu, 1997), and microstimulation of the SEF can elicit smooth pursuit eye movements (Tian and Lynch, 1995, 1996) and increase pursuit velocity (Missal and Heinen, 2001). In humans, neuroimaging studies consistently find greater activity in the SEF during pursuit than during fixation (Berman et al., 1999; Petit and Haxby, 1999; O'Driscoll et al., 2000). The SEF seems to be particularly important for pursuit initiation and pursuit at the target direction reversal. In non-human primates, microstimulation of the SEF 75-275 ms before pursuit initiation was found to increase initial pursuit velocity but to have no effect on pursuit maintenance (Missal and Heinen, 2001). During periodic pursuit, a ramp-like increase in neural activity in the SEF was observed across each half cycle, with neural activity at its highest point just before the target reverses direction (Heinen, 1994; Heinen and Liu, 1997). This suggests that the SEF may play a role in predicting the timing of the the same subject.
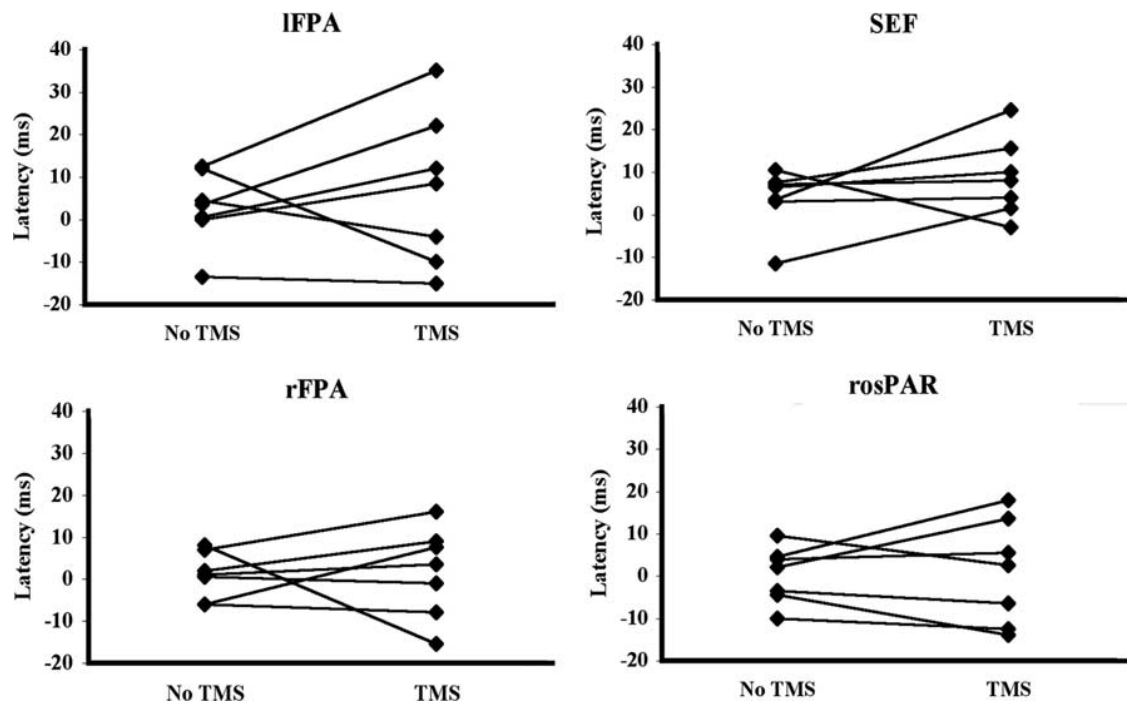

Figure 6. Experiment 2. Effect of TMS on the time required for the eye to reverse direction when applied at the target direction reversal. Each individual's direction reversal latency with and without TMS is connected by a straight line. There was no effect of TMS on direction reversal latency for any ROI and no interaction of TMS with direction (all $p>0.05$ ).

\begin{tabular}{lrrl}
\multicolumn{3}{l}{ Table 4. Experiment 3: results for the main effect of TMS } \\
\hline \\
\cline { 2 - 3 } Relocity $(\% / s)$ & & \\
\cline { 2 - 3 } Region & No TMS & \multicolumn{1}{c}{ TMS } & Average percentage change \\
\hline IFPA & & & \\
$\quad$ Peak velocity & $24.0(0.5)$ & $23.1(0.6)$ & $-4.2(0.8)^{* *}$ \\
$\quad$ Target turnaround & $3.6(0.1)$ & $5.0(0.6)$ & $+40.3(15.2)^{*}$ \\
leg-S1 & & & \\
$\quad$ Peak velocity & $23.9(0.4)$ & $23.1(0.8)$ & $-3.6(2.0)$ \\
$\quad$ Target turnaround & $3.8(0.1)$ & $4.3(0.3)$ & $+14.6(7.1)$ \\
\hline
\end{tabular}

Data are the mean (SE) of pursuit eye velocity. ${ }^{*} p<0.05 ;{ }^{* *} p<0.01$.

\section{Peak Target Velocity}
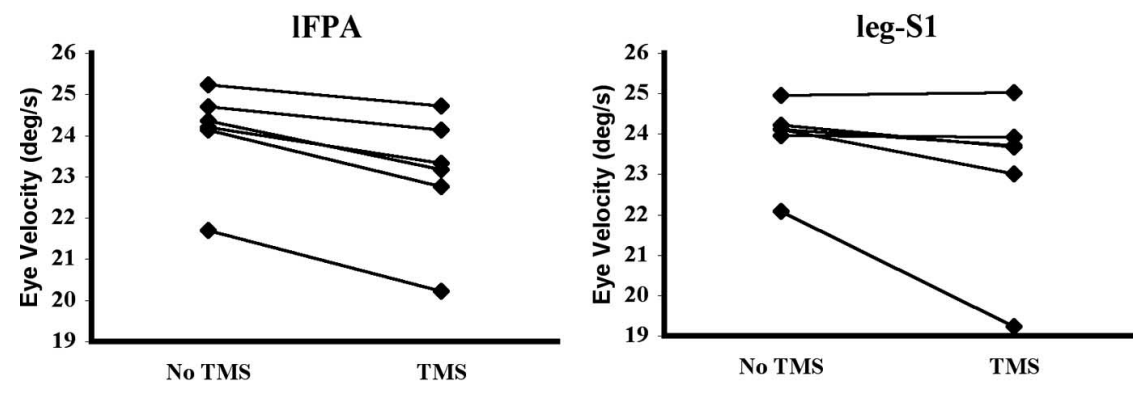

\section{Target Turnaround}
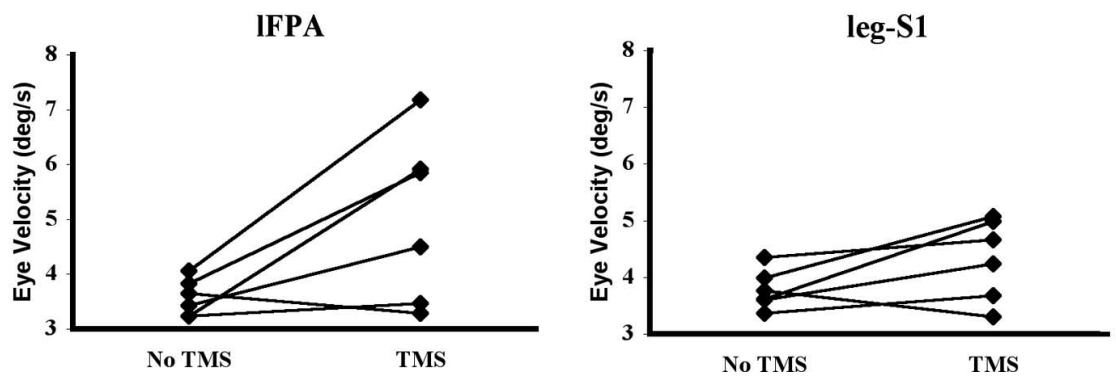

Figure 7. Experiment 3. Effect of TMS on eye velocity when applied to the IFPA and leg-S1 at peak target velocity and the target direction reversal. Each individual's eye velocity with and without TMS is connected by a straight line. Consistent with the stimulation results of the IFPA in both experiments 1 and 2, TMS applied at peak target velocity significantly decreased eye velocity and TMS applied at the target direction reversal significantly increased eye velocity. TMS applied to the leg-S1 at peak target velocity or the target direction reversal did not affect eye velocity $(p>0.05)$. The outlying trace in the two top panels belong to 
target turnaround (Heinen, 1994), and possibly in planning pursuit in the opposite direction (Krauzlis, 2004). Patients with dorsomedial lesions have been found to have an increased latency to reverse direction during pursuit of predictable targets (Heide et al., 1996), consistent with these hypotheses.

We applied TMS just before the target reversed direction, when SEF firing in monkeys is at its highest (Heinen and Liu, 1997), with the hypothesis that latency of the eye to reverse direction and the initial eye speed in the new direction would be affected. Eye velocity was significantly increased in the new direction during TMS cycles compared with control cycles and was not affected when stimulation was applied in mid-cycle. However, we found no effect on latency of direction reversal. It is possible that pursuit timing and velocity are controlled independently (Barnes and Donelan, 1999) and that the timing of stimulation used here, which did produce enhancement of eye velocity, may not have coincided with the optimal timing to produce changes in latency. Future studies might stimulate at multiple time points with respect to the turnaround to determine whether the latency to reverse direction can be modified with TMS.

\section{rosPAR}

The effect of stimulation of the rosPAR region was unexpected. This control region was intended to be the human homolog of macaque area AIP, a region in the most anterior portion of the intraparietal sulcus that has been implicated in reaching and grasping (Sakata et al., 1995). This region has not been implicated previously in smooth pursuit. Thus, explanations are necessarily speculative. However, the AIP has been reported to be involved in applying directional expectations to moving objects (Shulman et al., 1999), a function relevant to pursuit at the turnaround, where the effects were observed. Alternatively, the effects of stimulation of rosPAR may have been driven by current spread to the adjacent anterior region of parietal area $\mathrm{E}(\mathrm{PEa})$. Recently, this region in macaque has been described as having dense reciprocal connections to physiologically defined FPA (Stanton et al., 2005). Although there are few data on its role in pursuit, it has been postulated that PEa may be involved in the coordination of eye and head or eye and hand movements during tracking (Stanton et al., 2005).

\section{References}

Barnes GR, Donelan SF (1999) The remembered pursuit task: evidence for segregation of timing and velocity storage in predictive oculomotor control. Exp Brain Res 129:57-67.

Berman RA, Colby CL, Genovese CR, Voyvodic JT, Luna B, Thulborn KR, Sweeney JA (1999) Cortical networks subserving pursuit and saccadic eye movements in humans: an FMRI study. Hum Brain Mapp 8:209-225.

Bullmore ET, Brammer MJ, Williams SCR, Rabe-Hesketh S, Janot N, David AS, Mellers JDC, Howard R, Sham P (1996) Statistical methods of estimation and inference for functional MR image analysis. Magn Reson Med 35:261-277.

Cracco RQ, Cracco JB, Maccabee PJ, Amassian VE (1999) Cerebral function revealed by transcranial magnetic stimulation. J Neurosci Methods 86:209-219.

Culham JC, Kanwisher NG (2001) Neuroimaging of cognitive functions in human parietal cortex. Curr Opin Neurobiol 11:157-163.

Dallos PJ, Jones JW (1963) Learning behaviour of the eye fixation control system. IEEE Trans Autom Control AC 8:218-227.

Debaere F, Swinnen SP, Beatse E, Sunaert S, Van Hecke P, Duysens J (2001) Brain areas involved in interlimb coordination: a distributed network. NeuroImage 14:947-958.

Fink GR, Frackowiak RS, Pietrzyk U, Passingham RE (1997) Multiple nonprimary motor areas in the human cortex. J Neurophysiol 77:2164-2174.
Gagnon D, O’Driscoll GA, Petrides M, Pike GB (2002) The effect of spatial and temporal information on saccades and neural activity in oculomotor structures. Brain 125:123-139.

Gottlieb JP, Bruce CJ, MacAvoy MG (1993) Smooth eye movements elicited by microstimulation in the primate frontal eye field. J Neurophysiol 69:786-799.

Gottlieb JP, MacAvoy MG, Bruce CJ (1994) Neural responses related to smooth-pursuit eye movements and their correspondence with electrically elicited smooth eye movements in the primate frontal eye field. J Neurophysiol 72:1634-1653.

Grasse KL, Lisberger SG (1992) Analysis of a naturally occurring asymmetry in vertical smooth pursuit eye movements in a monkey. J Neurophysiol 67:164-179.

Grefkes C, Fink GR (2005) The functional organization of the intraparietal sulcus in humans and monkeys. J Anat 207:3-17.

Grosbras MH, Paus T (2002) Transcranial magnetic stimulation of the human frontal eye field: effects on visual perception and attention. J Cogn Neurosci 14:1109-1120.

Grosbras MH, Paus T (2003) Transcranial magnetic stimulation of the human frontal eye field facilitates visual awareness. Eur J Neurosci 18:3121-3126.

Grosbras MH, Lobel E, Van de Moortele PF, LeBihan D, Berthoz A (1999) An anatomical landmark for the supplementary eye fields in human revealed with functional magnetic resonance imaging. Cereb Cortex 9:705-711.

Heide W, Kurzidim K, Kompf D (1996) Deficits of smooth pursuit eye movements after frontal and parietal lesions. Brain 119:1951-1969.

Heinen SJ (1993) Evidence of a timing mechanism for predictive smooth pursuit in frontal cortex. In: Contemporary ocular motor and vestibular research: a tribute to David A. Robinson (Fuchs AF, Brandt T, Buttner U, Zee D, eds), pp 408-410. Stuttgart, Germany: New York Thieme.

Heinen SJ (1995) Single neuron activity in the dorsomedial frontal cortex during smooth pursuit eye movements. Exp Brain Res 104:357-361.

Heinen SJ, Liu M (1997) Single-neuron activity in the dorsomedial frontal cortex during smooth-pursuit eye movements to predictable target motion. Vis Neurosci 14:853-865.

Jahanshahi M, Rothwell J (2000) Transcranial magnetic stimulation studies of cognition: an emerging field. Exp Brain Res 131:1-9.

Kawashima R, Naitoh E, Matsumura M, Itoh H, Ono S, Satoh K, Gotoh R, Koyama M, Inoue K, Yoshioka S, Fukuda H (1996) Topographic representation in human intraparietal sulcus of reaching and saccade. NeuroReport 7:1253-1256.

Keating EG (1991) Frontal eye field lesions impair predictive and visuallyguided pursuit eye movements. Exp Brain Res 86:311-323.

Keating EG (1993) Lesions of the frontal eye field impair pursuit eye movements but preserve the predictions driving them. Behav Brain Res 53:91-104.

Keating EG, Pierre A (1996) Architecture of a gain controller in the pursuit system. Behav Brain Res 81:173-181.

Krauzlis RJ (2004) Recasting the smooth pursuit eye movement system. J Neurophysiol 91:591-603.

Leigh RJ, Zee DS (1999) The neurology of eye movements, Ed 3. New York: Oxford UP.

Lekwuwa GU, Barnes GR (1996) Cerebral control of eye movements. I. The relationship between cerebral lesion sites and smooth pursuit deficits. Brain 119:473-490.

Lisberger SG, Westbrook LE (1985) Properties of visual inputs that initiate horizontal smooth pursuit eye movements in monkeys. J Neurosci 5:1662-1673.

Lynch JC (1987) Frontal eye field lesions in monkeys disrupt visual pursuit. Exp Brain Res 68:437-441.

MacAvoy MG, Gottlieb JP, Bruce CJ (1991) Smooth pursuit eye movement representation in the primate frontal eye field. Cereb Cortex 1:95-102.

Missal M, Heinen SJ (2001) Facilitation of smooth pursuit initiation by electrical stimulation in the supplementary eye fields. J Neurophysiol 86:2413-2425.

Missal M, Heinen SJ (2004) Supplementary eye fields stimulation facilitates anticipatory pursuit. J Neurophysiol 92:1257-1262.

Morrow MJ, Sharpe JA (1995) Deficits of smooth-pursuit eye movement after unilateral frontal lobe lesions. Ann Neurol 37:443-451.

Muri RM, Hess CW, Meienberg O (1991) Transcranial stimulation of 
the human frontal eye field by magnetic pulses. Exp Brain Res $86: 219-223$.

Muri RM, Rivaud S, Vermersch AI, Leger JM, Pierrot-Deseilligny C (1995) Effects of transcranial magnetic stimulation over the region of the supplementary motor area during sequences of memory-guided saccades. Exp Brain Res 104:163-166.

O’Driscoll GA, Benkelfat C, Florencio PS, Wolff ALV, Joober R, Lal S, Evans AC (1999) Neural correlates of eye-tracking deficits in first-degree relatives of schizophrenic patients: a PET study. Arch Gen Psychiatry 56:1127-1134.

O’Driscoll GA, Wolff AL, Benkelfat C, Florencio PS, Lal S, Evans AC (2000) Functional neuroanatomy of smooth pursuit and predictive saccades. NeuroReport 11:1335-1340.

Ohtsuka K, Enoki T (1998) Transcranial magnetic stimulation over the posterior cerebellum during smooth pursuit eye movements in man. Brain 121:397-398.

Paus T (1996) Location and function of the human frontal eye-field: a selective review. Neuropsychologia 34:475-483.

Petit L, Haxby JV (1999) Functional anatomy of pursuit eye movements in humans as revealed by fMRI. J Neurophysiol 82:463-471.

Priori A, Bertolasi L, Rothwell JC, Day BL, Marsden CD (1993) Some saccadic eye movements can be delayed by transcranial magnetic stimulation of the cerebral cortex in man. Brain 116:355-367.

Robins LN, Helzer JE, Croughan J, Ratcliff KS (1981) National Institute of Mental Health diagnostic interview schedule. Its history, characteristics, and validity. Arch Gen Psychiatry 38:381-389.

Robinson D (1965) The mechanics of human smooth pursuit eye movement. J Physiol (Lond) 180:569-591.

Sakata H, Taira M, Murata A, Mine S (1995) Neural mechanisms of visual guidance of hand actions in the parietal cortex of the monkey. Cereb Cortex 5:429-438.

Schall JD (1991) Neuronal activity related to visually guided saccadic eye movements in the supplementary motor area of rhesus monkeys. J Neurophysiol 66:530-558.

Shulman GL, Ollinger JM, Akbudak E, Conturo TE, Snyder AZ, Petersen SE, Corbetta M (1999) Areas involved in encoding and applying directional expectations to moving objections. J Neurosci 19:8480-9496.

Stanton GB, Friedman HR, Dias EC, Bruce CJ (2005) Cortical afferents to the smooth-pursuit region of the macaque monkey's frontal eye field. Exp Brain Res 65:179-192.

Stark L, Vossius G, Young LR (1962) Predictive control of eye tracking movements. IRE Trans Hum Factors Electron HFE 3:52-57.

Stippich C, Ochmann H, Sartor K (2002) Somatotopic mapping of the human primary sensorimotor cortex during motor imagery and motor execution by functional magnetic resonance imaging. Neurosci Lett 331:50-54.

Tanaka M, Lisberger SG (2001) Regulation of the gain of visually guided smooth-pursuit eye movements by frontal cortex. Nature 409:191-194.

Tanaka M, Lisberger SG (2002) Enhancement of multiple components of pursuit eye movement by microstimulation in the arcuate frontal pursuit area in monkeys. J Neurophysiol 87:802-818.

Terao Y, Fukuda H, Ugawa Y, Hikosaka O, Hanajima R, Furubayashi T, Sakai K, Miyauchi S, Sasaki Y, Kanazawa I (1998) Visualization of the information flow through human oculomotor cortical regions by transcranial magnetic stimulation. J Neurophysiol 80:936-946.

Tian JR, Lynch JC (1995) Slow and saccadic eye movements evoked by microstimulation in the supplementary eye field of the cebus monkey. J Neurophysiol 74:2204-2210.

Tian JR, Lynch JC (1996) Functionally defined smooth and saccadic eye movement subregions in the frontal eye fields of Cebus monkeys. J Neurophysiol 76:2740-2753.

van den Berg AV (1988) Human smooth pursuit during transient perturbations of predictable and unpredictable target movement. Exp Brain Res 72:95-108.

Wessel K, Kompf D (1991) Transcranial magnetic brain stimulation: lack of oculomotor response. Exp Brain Res 86:216-218.

Worsley KJ, Marrett S, Neelin P, Vandal AC, Friston KJ, Evans AC (1996) A unified statistical approach for determining significant signals in images of cerebral activation. Hum Brain Mapp 4:58-73.

Worsley KJ, Liao C, Grabove M, Petre V, Ha B, Evans AC (2000) A general statistical analysis for fMRI data. NeuroImage 11:S648.

Yasui S, Young LR (1984) On the predictive control of foveal eye tracking and slow phases of optokinetic and vestibular nystagmus. J Physiol (Lond) 347:17-33.

Zangemeister W, Canavan A, Hoemberg V (1995) Frontal and parietal transcranial magnetic stimulation (TMS) disturbs programming of saccadic eye movements. J Neurol Sci 133:42-52. 